\title{
Circular RNA hsa_circ_105039 promotes cardiomyocyte differentiation by sponging miR-17 to regulate cyclinD2 expression
}

\author{
BOSHI YU, MENGMENG LI, SHU PING HAN, ZHANGBIN YU and JINGAI ZHU \\ Department of Pediatrics, Women's Hospital of Nanjing Medical University Nanjing \\ Maternity and Child Health Care Hospital, Nanjing, Jiangsu 210004, P.R. China
}

Received April 22, 2021; Accepted September 10, 2021

DOI: $10.3892 / \mathrm{mmr} .2021 .12501$

\begin{abstract}
Previously it was found that hsa_circ_105039 was underexpressed in the heart tissue of patients with congenital heart disease (CHD). However, the function and mechanism of hsa_circ_105039 in CHD are unclear. In the present study, induced pluripotent stem (iPS) cells were differentiated into cardiomyocytes using 1\% dimethyl sulfoxide (DMSO). Cell differentiation, viability, migration and apoptosis were measured before and following hsa_circ_105039 knockdown or overexpression. The results indicated that hsa_circ_105039 overexpression promoted cell differentiation, viability and migration; whereas apoptosis was simultaneously repressed. A luciferase reporter assay verified that hsa_circ_105039 acted as a sponge for microRNA (miR)-17 and that cyclinD2 was a direct target of miR-17. Furthermore, differentiation-related genes and proteins were analyzed by reverse transcription-quantitative PCR and western blotting, respectively. The results showed that hsa_circ_105039 could also upregulate the expression of differentiation-related genes and proteins, including natriuretic peptide A, cardiac troponin I, GATA-binding protein 4 and homobox transcription factor, in iPS cells. The results suggested that hsa_circ_105039 exerted a protective effect by promoting miR-17/cyclinD2 in DMSO-induced iPS
\end{abstract}

Correspondence to: Professor Zhangbin Yu or Dr Jingai Zhu, Department of Pediatrics, Women's Hospital of Nanjing Medical University Nanjing Maternity and Child Health Care Hospital, 23 Tian Fei Xiang, Mo Chou Road, Nanjing, Jiangsu 210004, P.R. China

E-mail: zhangbinyu@njmu.edu.cn

E-mail: zhujingai1983@163.com

Abbreviations: CHD, congenital heart disease; DMSO, dimethyl sulfoxide; iPS, induced pluripotent stem; ANP, atrial natriuretic peptide; $\alpha$-MHC, $\alpha$-myosin heavy chain; cTnI, cardiac troponin I; GAPDH, glyceraldehyde-3-phosphate dehydrogenase; GATA4, GATA-binding protein 4; MTT, 3-(4,5-dimethylthiazol-2-yl)-2,5-diphenyl-tetrazolium bromide; Nkx2.5, homobox transcription factor; RT-qPCR, reverse transcription-quantitative PCR

Key words: circular RNA, microRNA-17, congenital heart disease cardiomyocytes, which indicated that hsa_circ_105039 is a potential key molecule for the diagnosis of CHD.

\section{Introduction}

Congenital heart disease (CHD) is a congenital disease with a high incidence, particularly in America, that has gradually increased in recent years $(1,2)$. CHD is the leading cause of infant mortality due to birth defects and accounts for nearly one-third of all congenital anomalies $(3,4)$. CHD is often accompanied by other complications and patients require repeated surgical procedures. This seriously affects the quality of life of patients and is also a burden on the family and society (5). Therefore, early prenatal diagnosis is important to investigate the occurrence of CHD and improve the medical management of mother and fetal outcomes $(6,7)$. In current obstetric care, the clinical strategy for diagnosing CHD mainly relies on fetal echocardiography in the second trimester of pregnancy $(8,9)$. However, only $\sim 50 \%$ of CHDs can be detected by echocardiography, which means that not all CHDs are detected during pregnancy (10). Hence, finding potential biomarkers for strict fetal CHD screening would contribute greatly to prenatal diagnosis.

Circular RNAs (circRNAs) have been identified as novel noncoding RNAs (ncRNAs) that are formed during RNA maturation through a process called splicing $(11,12)$. CircRNAs have a closed-loop structure without $3^{\prime}$ and $5^{\prime}$ polarity (13). CircRNAs are cyclic in structure and contain introns of gene fragments (14). Studies have shown that microRNAs (miRNAs) are sponged by circRNAs (15-17). Previous studies have shown that circRNAs can bind miRNAs as competitive endogenous RNAs (ceRNAs), preventing miRNAs from binding their target mRNAs and resulting in the increased expression of targets. This regulatory mechanism is the most common mechanism for circRNAs (18). Studies have shown that the circRNA levels in the heart are diverse and closely associated with heart disease $(19,20)$.

Our previous study found that hsa_circ_105039 is significantly downregulated in CHD (21). However, how hsa_circ_105039 activity regulates CHD disease progression remains to be elucidated. The current study was designed to determine the function of the circRNA hsa_circ_105039 in the pathogenesis of CHD, as well as to search for its target miRNA. 


\section{Materials and methods}

Cell culture and differentiation. The human induced pluripotent stem (iPS) cell line (provided by The Cell Bank of Type Culture Collection of The Chinese Academy of Sciences; cat. no. SCSP-1302) was cultured in mTeSR1 media (Stemcell Technologies, Inc.) at $37^{\circ} \mathrm{C}$ with $5 \% \mathrm{CO}_{2}$. Following the digestion of iPS cells with trypsin, the concentration was adjusted to 10 cells $/ \mu 1$ and then placed at the bottom of the culture dish at $40 \mu 1$ per drop. The cells were returned to the incubator to be used for hanging drop culture. iPS cells were seeded into $0.1 \%$ gelatin-coated petri dishes to remove murine embryonic fibroblasts. At 2 days following inoculation, the iPS cells were gently aspirated. The cells were seeded in a low-adhesion petri dish and cultured for 5 days to induce embryoid body (EB) formation. The culture conditions were DMEM (cat. no. 11960069; Gibco; Thermo Fisher Scientific, Inc.), 10\% fetal bovine serum (cat.no. 16140071; Gibco; Thermo Fisher Scientific, Inc.), 1\% L-glutamine (cat. no. 25030081; Gibco; Thermo Fisher Scientific, Inc.) and 1\% penicillin-streptomycin (cat. no. SV30010; HyClone; Cytiva). After inducing the formation of $\mathrm{EB}$, the cells were inoculated into gelatin-coated petri dishes for culture. DMSO (1\%) was added to induce induction on days 1,3 and 5 of culture and myocardial differentiation medium (cat. no. 5911; ScienCell Research Laboratories, Inc.) was added 1 week following induction.

Cell transfection. Small interfering (si)RNAs, negative controls and miR-17 inhibitors were synthesized by Sangon Biotech Co., Ltd. DMSO-induced cardiomyocytes were transfected with Lipofectamine ${ }^{\circledR} 3000$ (Invitrogen; Thermo Fisher Scientific, Inc.) according to the manufacturer's instructions. Briefly, $100 \mathrm{nM}$ siRNA was transfected into the DMSO-induced cardiomyocytes at $37^{\circ} \mathrm{C}$ with $5 \% \mathrm{CO}_{2}$. After incubation for $6 \mathrm{~h}$, the medium was replaced with the mTeSR 1 followed by incubation at $37^{\circ} \mathrm{C}$ with $5 \% \mathrm{CO}_{2}$ for $48 \mathrm{~h}$, and transfection efficiency was measured via reverse transcription-quantitative PCR (RT-qPCR). The target sequences of si-negative control (NC) and si-hsa_circ_105039 were as follows: si-NC, 5'-GACCGGCAGATCGAAACTA AA-3' and si-hsa_circ_105039, 5'-GCAAGACAGCAAGCAG ATCTA-3'.

Cell viabilityassay. A3-(4,5-dimethyl-2-thiazolyl)-2,5-diphenyl-2-H-tetrazolium bromide (MTT; Sigma-Aldrich; Merck $\mathrm{KGaA}$ ) assay was used to evaluate cytotoxic activity. The iPS cells were induced into cardiomyocytes using $1 \%$ DMSO, and the resulting cells were transfected with the si-RNAs and cultured at $37^{\circ} \mathrm{C}$ with $5 \% \mathrm{CO}_{2}$ for $48 \mathrm{~h}$. The cells were collected and cultured in 96-well plates at a concentration of 5,000 cells/well. Following incubation at $37^{\circ} \mathrm{C}$ with $5 \% \mathrm{CO}_{2}$ for $24 \mathrm{~h}$, the media was aspirated, $20 \mu 15 \mathrm{mg} / \mathrm{ml}$ MTT was added to each well and the cells were incubated at $37^{\circ} \mathrm{C}$ with $5 \% \mathrm{CO}_{2}$ for another $4 \mathrm{~h}$. Then, the supernatant was completely replaced with $150 \mu \mathrm{l}$ DMSO to dissolve the purple crystals. Finally, following $10 \mathrm{~min}$ of agitation, the absorbance was measured at $570 \mathrm{~nm}$.

Transwell assay. The transfected cells were cultured in a six-well plate until confluence reached $\sim 100 \%$ and then
$1 \%$ fetal bovine serum was added to the culture medium. The DMSO-induced cardiomyocytes were adjusted to $7 \times 10^{4}$ and the cells were seeded in a 24-well Transwell chamber (pore size, $8 \mu \mathrm{m}$; cat. no. 3422; Corning, Inc.). At $24 \mathrm{~h}$ following transfection, $5 \times 10^{4}$ iPS cells were inoculated into the upper compartment of serum-free medium and $10 \%$ fetal bovine serum was added to the lower compartment. Following culture at $37^{\circ} \mathrm{C}$ for $8 \mathrm{~h}$, the bottom cells were fixed with $4 \%$ paraformaldehyde at room temperature for $10 \mathrm{~min}$ and then stained with $400 \mu 10.4 \%$ crystal violet (Beijing Solarbio Science \& Technology Co., Ltd.) at room temperature for $10 \mathrm{~min}$ and images were acquired under an inverted microscope (Ts2R; Nikon Corporation) at $\times 200$ magnification, and then the number of cells stained positive for crystal violet were counted.

Flow cytometry analysis. Cells were washed three times with phosphate buffer. Then, $1 \times 10^{6}$ cells were resuspended in $100 \mu 1$ with $5 \mu 1$ FITC-Annexin V (eBioscience; Thermo Fisher Scientific, Inc.) and propidium iodide (eBioscience; Thermo Fisher Scientific, Inc.) antibodies in the dark. Then, the cells were incubated at $4^{\circ} \mathrm{C}$ for $30 \mathrm{~min}$. After washing three times with PBS, the cells were analyzed by flow cytometry (Accuri $^{\text {TM }}$ C6; BD Biosciences) using FlowJo version 10 software (FlowJo LLC) (22). The percentage of late apoptotic cells was calculated.

Luciferase reporter assay. PsiCHECK-circ_105039-wild-type (WT) and mutated (MUT) were obtained from Sangon Biotech Co., Ltd. miR-17 mimic and miR-17-NC were synthesized by Sangon Biotech Co., Ltd. IPS cells were cultured in mTeSR1 media. The sequences of psiCHECK-circ_105039-WT/MUT were cloned into the pGL3-basic reporter (Promega Corporation). For the reporter assays, cells were co-transfected with pRT-TK Renilla plasmid (cat. no. E2241; Promega Corporation) and pGL3 -circ_105039-WT/MUT firefly luciferase reporter plasmids as well as miR-17 mimic or control using Lipofectamine ${ }^{\circledR} 3000$ (Invitrogen; Thermo Fisher Scientific, Inc.). After culturing for $48 \mathrm{~h}$, the luciferase activity was measured using the dual luciferase reporter gene assay system (GloMax 20/20; Promega Corporation) according to the manufacturer's instructions. The sequences of miRNA mimics were as follows: control sense, 5'-UUCUCCGAACG UGUCACGUTT-3' and control anti-sense, 5'-ACGUGACAC GUUCGGAGAATT-3'; miR-17 mimic sense, 5'-CAAAGU GCUUACAGUGCAGGUAG-3' and anti-sense, 5'-ACCUGC ACUGUAAGCACUUUGUU-3'. The firefly luciferase activity was normalized to Renilla luciferase activity for each sample. The data were recorded with a SpectraMax M5e microplate reader (Molecular Devices, LLC).

$R T$-qPCR. mRNA was isolated from DMSO-induced cardiomyocytes $\left(2 \times 10^{5}\right.$ cells) cells by TRIzol Plus ${ }^{\circledR}$ reagent (cat. no. 9108; Takara Biotechnology Co., Ltd.). Total cDNA was reverse transcribed using PrimeScript ${ }^{\mathrm{TM}}$ RT reagent kit with gDNA Eraser (cat. no. RR047A; Takara Biotechnology Co., Ltd.) according to the manufacturer's protocols. RT-qPCR was performed with the SYBR-Green PCR kit (cat. no. RR820; Takara Biotechnology Co., Ltd.) on a 7900HT Fast Real-Time PCR System (Applied Biosystems; Thermo Fisher Scientific, Inc.). Thermocycling conditions were as follows: $95^{\circ} \mathrm{C}$ for 
Table I. Primer sequences used in reverse transcription-quantitative PCR.

\begin{tabular}{llll}
\hline Gene & \multicolumn{1}{c}{ Forward sequences $\left(5^{\prime}-3^{\prime}\right)$} & \multicolumn{1}{c}{ Reverse sequences $\left(5^{\prime}-3^{\prime}\right)$} & Size, bp \\
\hline 18S rRNA & CTACCACATCCAAGGAAGCA & TTTTTCGTCACTACCTCCCCG \\
circ_079265 & CGTCGCCCACATAGGAATC & ACTGACTTGAGACCAGTTGAATAA \\
GAPDH & CAGGGCTGCTTTTAACTCTGGTAA & GGGTGGAATCATATTGGAACATGT & 91 \\
Nkx2.5 & GCAGGCGCAGGTCTATG & TGCGTGGACGTGAGTTTC & 101 \\
GATA4 & ATGGGACGGGTCACTATCT & GCGCTGAGGCTTGATGA & 105 \\
$\alpha-$ MHC & CCCGCTTTGGGAAATTCATTAG & CTCCAGCAGGTAGGTCTCTAT & 86 \\
ANP & ACGCAGACCTGATGGATTTC & GCTTCTTCATTCGGCTCACT & 109 \\
cTnI & GACAAGGTGGATGAAGAGAGATAC & CTTGCCTCGAAGGTCAAAGA & 102
\end{tabular}

circ, circular RNA; Nkx2.5, homobox transcription factor; GATA4, GATA-binding protein 4; $\alpha$-MHC, $\alpha$-myosin heavy chain; ANP, atrial natriuretic peptide; cTnI, cardiac troponin I.

$30 \mathrm{sec}$ and 40 cycles of $95^{\circ} \mathrm{C}$ for $15 \mathrm{sec}, 60^{\circ} \mathrm{C}$ for $60 \mathrm{sec}$; and melt curves were recorded with $95^{\circ} \mathrm{C}$ for $15 \mathrm{sec}, 72^{\circ} \mathrm{C}$ for $60 \mathrm{sec}$ and $95^{\circ} \mathrm{C}$ for $15 \mathrm{sec}$. Changes in expression were calculated using the $2^{-\Delta \Delta \mathrm{Cq}}$ method (23). The results were expressed as the ratio of optimal density to glyceraldehyde-3-phosphate dehydrogenase (GAPDH). Each experiment was performed independently three times. Primer sequences are listed in Table I.

Western blotting. Total protein samples were extracted from cells lysed with RIPA lysis buffer (Beyotime Institute of Biotechnology). The samples were then analysed by western blotting. The proteins were collected and centrifuged at $12,000 \mathrm{x} \mathrm{g}$ for $15 \mathrm{~min}$ at $4^{\circ} \mathrm{C}$. A BCA protein assay kit (Beyotime Institute of Biotechnology) was used to determine protein concentration. The samples $(20 \mu \mathrm{g} /$ lane $)$ were separated via SDS-PAGE $[12 \%$ for atrial natriuretic peptide (ANP) and cardiac troponin I (cTnI); 10\% for cyclinD2, GATA-binding protein 4 (GATA4), homobox transcription factor $(\mathrm{Nkx} 2.5)$ and GAPDH; $6 \%$ for $\alpha$-myosin heavy chain $(\alpha-\mathrm{MHC})]$ and transferred onto a polyvinylidene fluoride (PVDF, $0.22 \mu \mathrm{m}$; cat. no. ISEQ00010; MilliporeSigma). The membranes were incubated in a blocking solution containing 5\% non-fat milk in PBS with $1 \%$ Tween-20 at room temperature for $1.5 \mathrm{~h}$ and then incubated with the primary antibodies diluted in blocking solution individually. The primary antibodies used were as follows: ANP (1:1,000; cat. no. ab190001; Abcam), cyclinD2 (1:2,000; cat. no. ab230883; Abcam), cTnI (1:1,000; cat. no. ab209809; Abcam), GATA4 (1:1,000; cat. no. ab84593; Abcam), Nkx2.5 (1:1,000; cat. no. ab106923; Abcam), $\alpha$-MHC (1:2,000; cat. no. ab185967; Abcam) and GAPDH (1:5,000; cat. no. ab181603; Abcam). After incubation with the primary antibodies at $4^{\circ} \mathrm{C}$ for $12 \mathrm{~h}$, the membranes were incubated with horseradish peroxidase-conjugated goat anti-rabbit IgG secondary antibody (1:10,000; cat. no. A0208; Beyotime Institute of Biotechnology) for $1 \mathrm{~h}$ at room temperature. The bound antibodies were detected using a chemiluminescence system (cat. no. 180-5001; Tanon Science and Technology Co., Ltd.). GAPDH was used as an internal control. Image Lab version 3.0 (Bio-Rad Laboratories, Inc.) and Quantity One version 4.62 (Bio-Rad Laboratories, Inc.) software were used for densitometry.
Bioinformatics analysis. The regulatory network of hsa_circ_105039 was evaluated by bioinformatics analysis (RegRNA 2.0 version, http://regrna2.mbc.nctu.edu.tw). To elucidate the potential function of miR-17, a bioinformatics analysis was performed using miRTarBase 7.0 version (http://mirtarbase.mbc.nctu.edu.tw/php/index.php) and TargetScan (http://www.targetscan.org/vert_72/).

Statistical analysis. All experimental data are shown as the mean \pm standard deviation. The experiments were performed independently in triplicate. Statistical graphs were made by GraphPad Prism 8.3 software (GraphPad Software, Inc.). Differences between experimental groups were analyzed for statistical significance using one-way analysis of variance followed by the Tukey's post hoc multiple comparison test. $\mathrm{P}<0.05$ was considered to indicate a significant difference.

\section{Results}

Hsa_circ_105039 knockdown suppresses viability, enhances apoptosis and promotes migration of iPS cells in vitro. Our previous study reported that hsa_circ_105039 was expressed at lower levels in the heart tissue of patients with CHD (21). To determine whether hsa_circ_105039 has a positive effect in promoting the viability of iPS cells, siRNA and overexpression plasmids targeting hsa_circ_105039 were used to knockdown and overexpress hsa_circ_105039 expression levels. Following iPS cell differentiation by DMSO, the gene expression of hsa_circ_105039 was significantly upregulated (Fig. 1A). When the plasmid was transfected for $48 \mathrm{~h}$, the expression of hsa_circ_105039 was analyzed by RT-qPCR (Fig. 1B). The MTT assay suggested that iPS cell viability was significantly promoted when hsa_circ_105039 was overexpressed, but was suppressed when hsa_circ_105039 was knocked down (Fig. 1C). Flow cytometry showed that the apoptosis of iPS cells was induced following hsa_circ_105039 knockdown (Fig. 1D and E). However, it was significantly suppressed when hsa_circ_105039 was overexpressed (Fig. 1D and E). In addition, the migration of iPS cells was suppressed after hsa_circ_105039 was knocked down (Fig. 1F and G). By contrast, it was promoted when hsa_circ_105039 was overexpressed (Fig. 1F and G). 
A

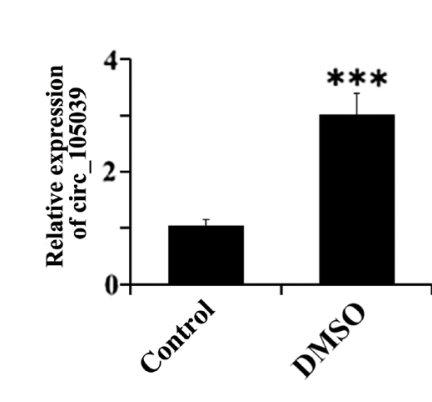

D

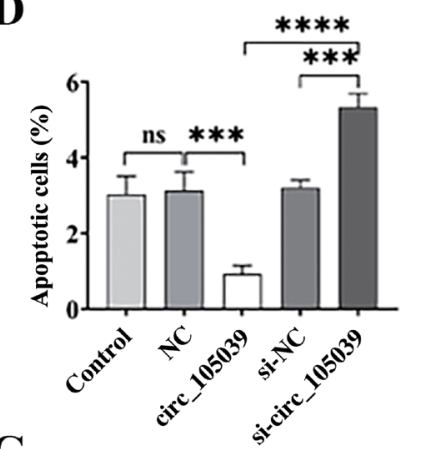

B

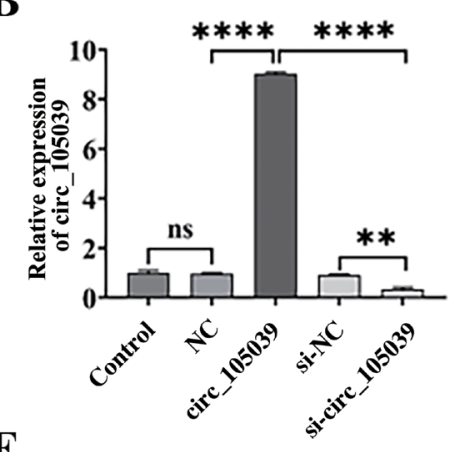

E

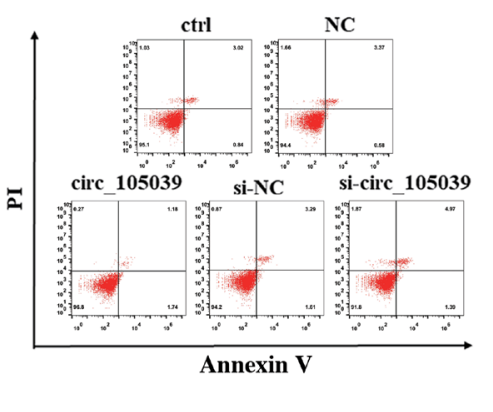

C
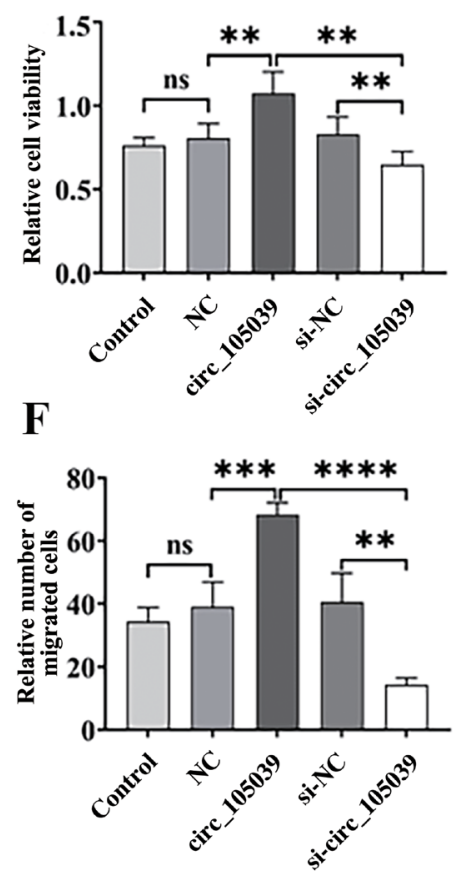

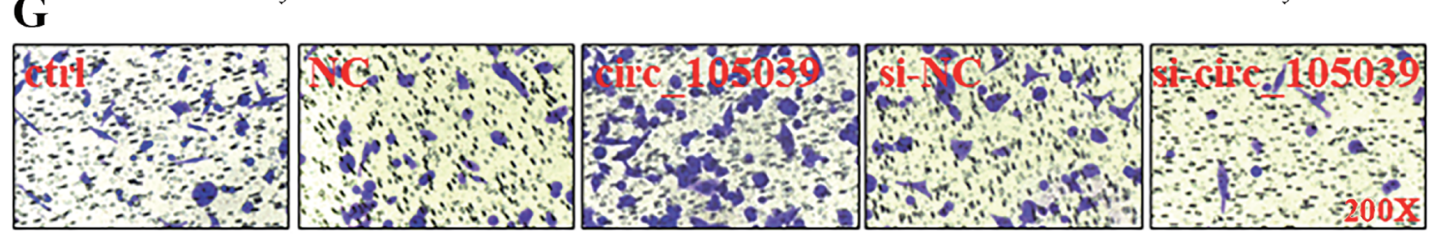

Figure 1. Expression of hsa_circ_105039 and cell viability, migration and apoptosis in DMSO-induced iPS cardiomyocytes. (A and B) Reverse transcription-quantitative PCR assay for the expression of hsa_circ_105039. (C) 3-(4,5-dimethyl-2-thiazolyl)-2,5-diphenyl-2-H-tetrazolium bromide assay of viability of iPS cells. (D) Statistical analysis histogram of flow cytometry. (E) Scatter plot of flow cytometry. (F and G) Transwell assay of iPS cell migration. n=3. ${ }^{* *} \mathrm{P}<0.01,{ }^{* * *} \mathrm{P}<0.005$ and ${ }^{* * * *} \mathrm{P}<0.001$. ns, not significant; iPS, induced pluripotent stem; DMSO, dimethyl sulfoxide; NC, negative control; circ, circular RNA; si, small interfering RNA.

Hsa_circ_105039 knockdown suppresses the expression of differentiation-related genes and proteins in iPS cells. To verify the role of hsa_circ_105039 in regulating iPS cell differentiation, the gene and protein expression levels of ANP, Nkx2.5, cTnI, $\alpha$-MHC and GATA4 were detected by RT-qPCR and western blotting, respectively (Fig. 2A-F). Following iPS cell differentiation by DMSO, the gene expression of ANP (Fig. 2B), Nkx2.5 (Fig. 2C), cTnI (Fig. 2D), $\alpha$-MHC (Fig. 2E) and GATA4 (Fig. 2F) was significantly increased following hsa_circ_105039 overexpression, but decreased when hsa_circ_105039 was knocked down. Western blot analysis showed that the protein levels of ANP, Nkx2.5, cTnI, $\alpha-\mathrm{MHC}$ and GATA4 were also increased or decreased following hsa_circ_105039 overexpression or knockdown, respectively (Fig. 2G-L). The results indicated that hsa_circ_105039 had a positive effect on the differentiation of iPS cells.

Hsa_circ_105039 directly binds to miR-17. It is known that circRNAs act as competing endogenous RNAs by sponging miRNAs (15-17). Thus, the regulatory network of hsa_circ_105039 was evaluated by bioinformatics analysis (RegRNA2.0). The results showed that miRNA-17 had a potential binding site for hsa_circ_105039 (Fig.3A). Therefore, in iPS cells co-transfected with WT hsa_circ_105039 and miR-17 mimics, the relative intensity of luciferase decreased
(Fig. 3B). In addition, the expression of miR-17 in iPS cells was detected by RT-qPCR. The results showed that the expression of miR-17 decreased when the hsa_circ_105039 gene was overexpressed (Fig. 3C). By contrast, the expression of miR-17 increased when the hsa_circ_105039 gene was knocked down (Fig. 3C). These results indicated that hsa_circ_105039 had a negative effect on miR-17.

miR-17 targets cyclinD2. To elucidate the potential function of miR-17, a bioinformatics analysis was performed using miRTarBase and TargetScan and a potential target gene of miR-17, cyclinD2, was identified. The 3'-UTR sequences of cyclinD2 mRNA were complementary to the miR-17 seed sequence (Fig. 4B). Plasmids containing the WT or MUT form of the 3'-UTR of cyclinD2 were constructed and expressed into iPS cells together with miR-con (NC group) or miR-17 mimics separately and a quantitative luciferase reporter was conducted. As shown in Fig. 4C, the relative intensity of luciferase activity was reduced in iPS cells co-transfected with WT hsa_circ_105039, but not in cells co-transfected with miR-17 mimics.

Hsa_circ_105039 regulates the differentiation of iPS cells by inhibiting miR-17. In addition, the viability of iPS cells following hsa_circ_105039 overexpression or knockdown 
A

1. Control

2. DMSO

3. NC+DMSO

4. circ_105039+DMSO

5. si-NC+DMSO

6. si-circ_105039+DMSO
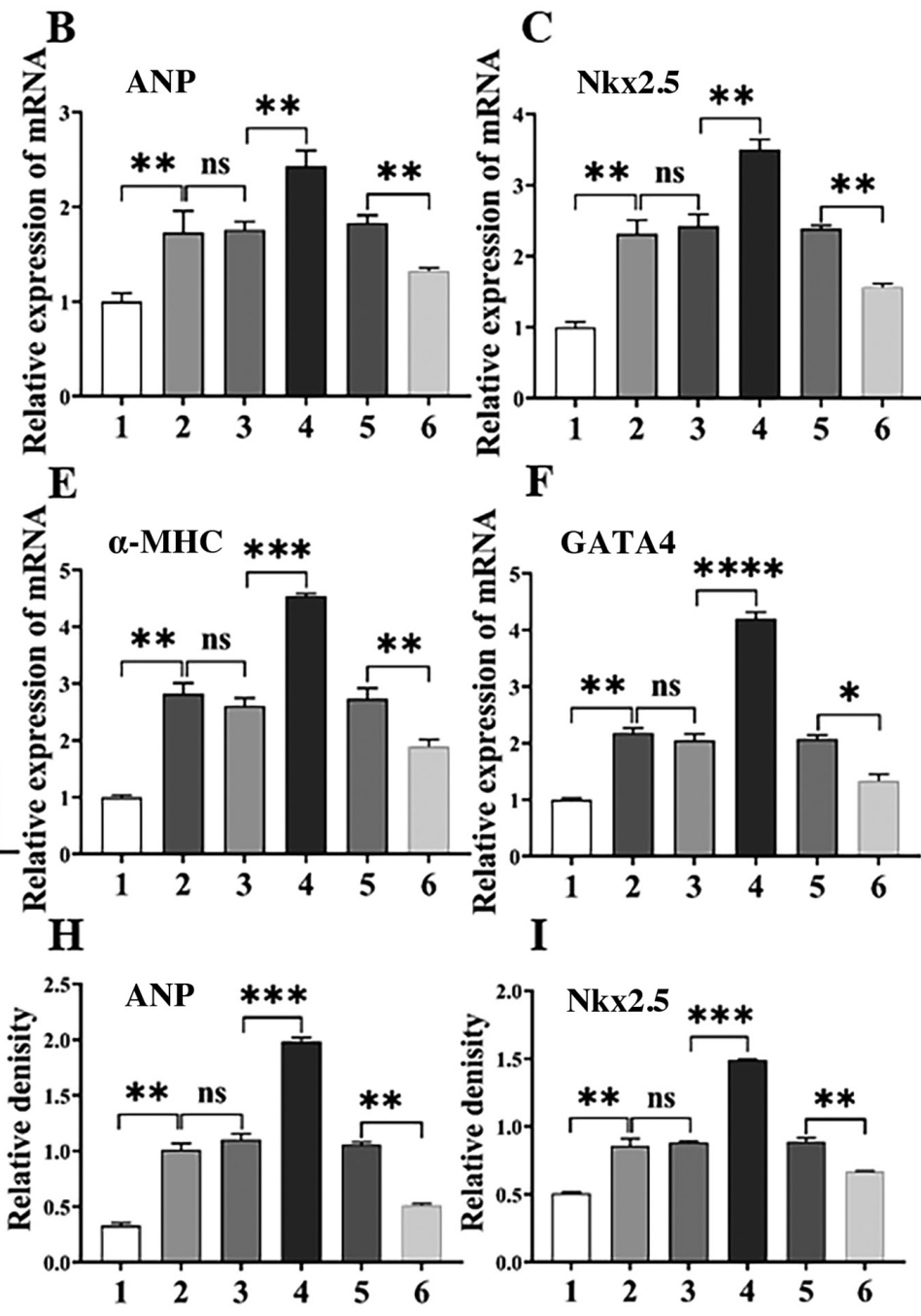

GAPDH $-\cdots-\cdots-\cdots$
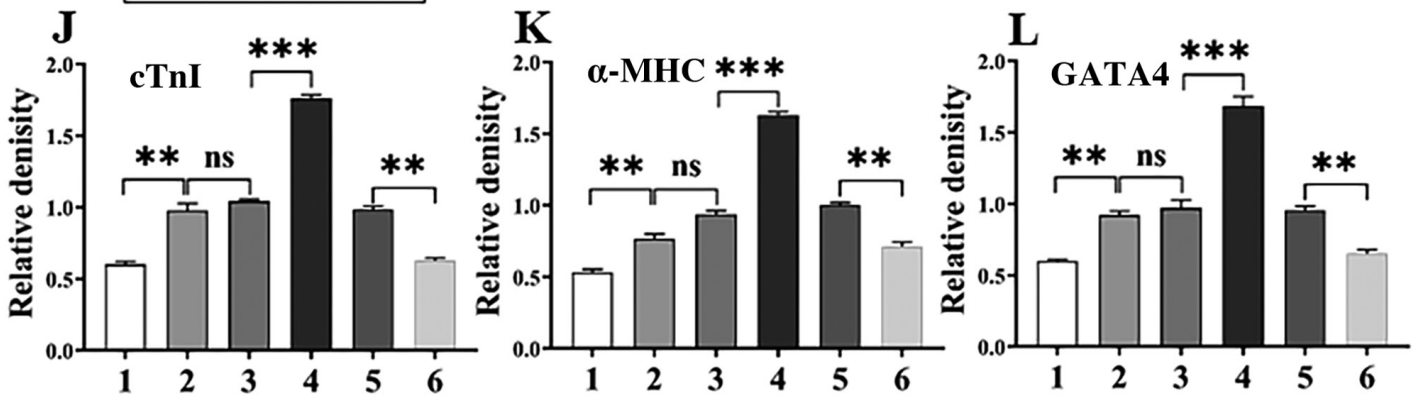

Figure 2. DMSO induces iPS cells to differentiate into cardiomyocytes and express differentiation-related genes and proteins in iPS cells. (A) Experimental grouping. Reverse transcription-quantitative PCR assay for the expression of (B) ANP, (C) Nkx2.5, (D) cTnI, (E) $\alpha$-MHC and (F) GATA4. (G) Western blotting assay for (H) ANP, (I) Nkx2.5, (J) cTnI, (K) $\alpha$-MHC and (L) GATA4. n=3. ${ }^{*} \mathrm{P}<0.05,{ }^{* * *} \mathrm{P}<0.01,{ }^{* * *} \mathrm{P}<0.005$ and ${ }^{* * * *} \mathrm{P}<0.001$. ns, not significant; iPS, induced pluripotent stem; DMSO, dimethyl sulfoxide; NC, negative control; circ, circular RNA; si, small interfering RNA; ANP, atrial natriuretic peptide; Nkx2.5, homobox transcription factor; cTnI, cardiac troponin I; $\alpha$-MHC, $\alpha$-myosin heavy chain; GATA4, GATA-binding protein 4.

(Fig. 4A) was analyzed and the results are shown in Fig. 4D and E. Compared with the control group, the viability of iPS cells was suppressed when hsa_circ_105039 was knocked down (Fig. 4D) in the si-circ_105039 + mimic group. However, compared with the $\mathrm{NC}+$ mimic group, the viability of iPS cells was promoted when hsa_circ_105039 was overexpressed in the circ_105039+ mimic group (Fig. 4E). These results indicated that hsa_circ_105039 promoted iPS cell viability by inhibiting miR-17.

To investigate whether hsa_circ_105039 regulated the differentiation of iPS cells by inhibiting miR-17, hsa_circ_105039 mimic and miR-17 inhibitor were co-transfected into the iPS cells. RT-qPCR results showed that the gene expression of cyclinD2 (Fig. 4F and G), ANP (Fig. 4H and I) and cTnI (Fig. 4J and K) were decreased in the si-circ_105039+ mimic group and recovered in the circ_105039 + mimic inhibitor group compared with the control group. In addition, western blotting (Fig. 5A and B) was performed to determine the protein expression of cyclinD2 (Fig. 5C and D), ANP (Fig. 5E and F) and cTnI (Fig. 5G and $\mathrm{H}$ ), the results were consistent with the results of the RT-qPCR assay. Altogether, these results indicated that 
A

5' - tggagaatgaggacgCGUGAAAc -3' circ_105039 MUT

\section{3'- gauggacgugacauuCGUGAAAc -5’ hsa-miR-17 | | | | | | | \\ 5' - tggagaatgaggacgGCACTTTc $-3^{\prime}$ circ_105039 WT}

B

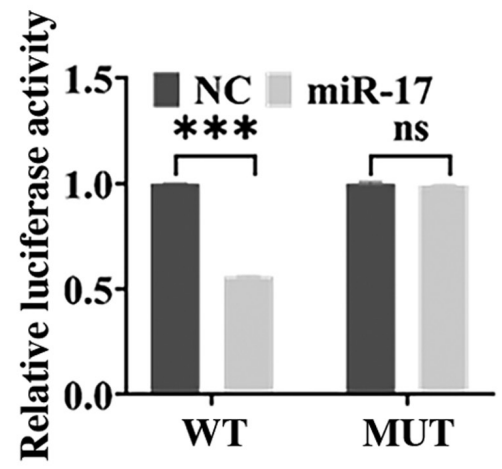

C

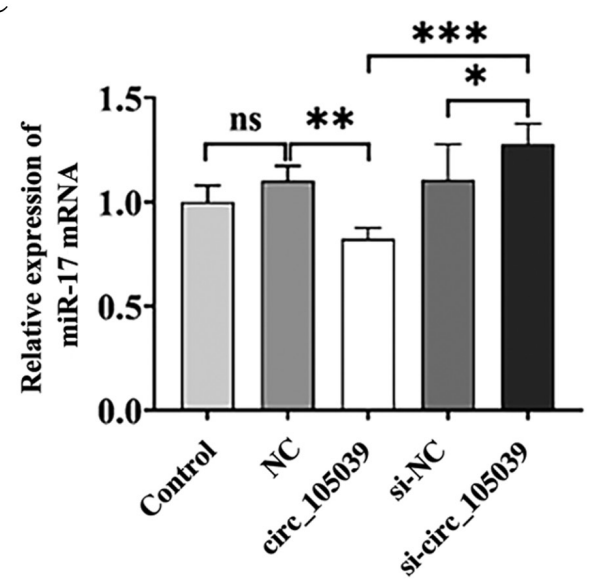

Figure 3. Hsa_circ_105039 acts as a sponge for miR-17. (A) Sequence alignment of hsa_circ_105039 and the potential binding site in the 3'-UTR of miR-17. (B) A luciferase reporter assay was performed in iPS cells co-transfected with hsa_circ_105039-WT plasmid or hsa_circ_105039-MUT plasmid.(C) Detection of miR-17 expression by reverse transcription-quantitative PCR. $\mathrm{n}=3$. ${ }^{*} \mathrm{P}<0.05,{ }^{* *} \mathrm{P}<0.01$ and ${ }^{* * *} \mathrm{P}<0.005$. ns, not significant; circ, circular $\mathrm{RNA}$; miR, micro RNA; si, small interfering RNA; iPS, induced pluripotent stem; WT, wild-type; MUT, mutated; NC, negative control.

hsa_circ_105039 promoted iPS cell viability and suppressed cell apoptosis by regulating the expression of cyclinD2 by inhibiting miR-17.

\section{Discussion}

IPS cells have characteristics similar to embryonic stem cells (24). They have the ability to differentiate into a number of types of cells (including cardiomyocytes). Cardiomyocytes derived from iPS cells recapitulate the phenotypic differences caused by genetic variation, making it an attractive human disease model (25). In addition, cardiomyocytes derived from iPS cells can also be used as source cells for heart regeneration in animal models. DMSO has both hydrophilic and lipophilic properties. It is a commonly used solvent for water-insoluble substances (26). DMSO has a great impact on cell cycle and cell apoptosis, can change cell metabolism process and thus affect cell growth (27). The differentiation of iPS cells into cardiomyocytes has three stages: First differentiation into mesoderm cells, then cardiomyocyte progenitor cells and finally mature cardiomyocytes (28). Most of the available protocols for using DMSO as a cardiogenic drug focus on its use in the first few days of EB culture (29). Studies have confirmed that DMSO can improve the efficiency of cardiomyocyte differentiation (27,30). However, the underlying mechanism of this molecular action is unclear. The present study chose DMSO (1\%) to induce iPS cell differentiation into cardiomyocytes. The gene and protein expression of ANP, Nkx2.5, cTnI, $\alpha-\mathrm{MHC}$ and GATA4 was detected to verify whether the differentiation was successful. ANP, Nkx2.5, cTnI, $\alpha$-MHC and GATA4 serve an important role in the maturation of cardiomyocytes. Cardiac contractile proteins (cTnI, $\alpha$-MHC and ANP) are specific markers marking the differentiation and maturation of cardiomyocytes and the transcription factors Nkx2.5 and GATA4 are early markers of the cardiac lineage and expressed throughout the duration of cardiac development (31-33). Aberrant expression of these specific molecular markers (cTnI, ANP, $\alpha$-MHC, Nkx2.5 and GATA4), including upregulation and downregulation, will contribute to abnormal cardiac cell differentiation and further result in failure of the early primitive heart tube formation, cardiomyocyte differentiation and heart morphogenesis (33-35).

CircRNAs serve a key role in a number of types of biological processes. However, initially, these molecules were only deemed to be missplicing products or intermediates of transcriptional processes and were not given much attention. Studies have shown that circRNAs can regulate the function of miRNAs by acting as miRNA sponges $(12,36)$. Our previous study discovered that hsa_circ_105039 was underexpressed in the heart tissues of patients with CHD (21). To the best of the authors' knowledge, this is the first time that hsa_circ_105039 has been reported in CHD. The present study analyzed the potentialfunction of hsa_circ_105039in the viability,migration and apoptosis of iPS cells. It was shown that hsa_circ_105039 was a mediator in the viability, migration and apoptosis of iPS cells. In addition, it was found that hsa_circ_105039 acted as a sponge for miR-17, which could be regulated in this biological process. miRNAs possess positive effects on the progression of CHD (37) but potentially act as sensitizing agents in breast cancer (38), tumorigenesis (39) and chronic pancreatitis (40). By performing software prediction analysis and luciferase reporter assays, the present study further found that miR-17 targeted cyclinD2. The miRNA target prediction 
A

\section{Control}

2. si-NC+mimic

3. si-NC+inhibitor

4. si-circ_105039+mimic

5. si-circ_105039+inhibitor

6. $\mathrm{NC}+\mathrm{mimic}$

7. NC+inhibitor

8. circ_105039+mimic

9. circ_105039+inhibitor

C
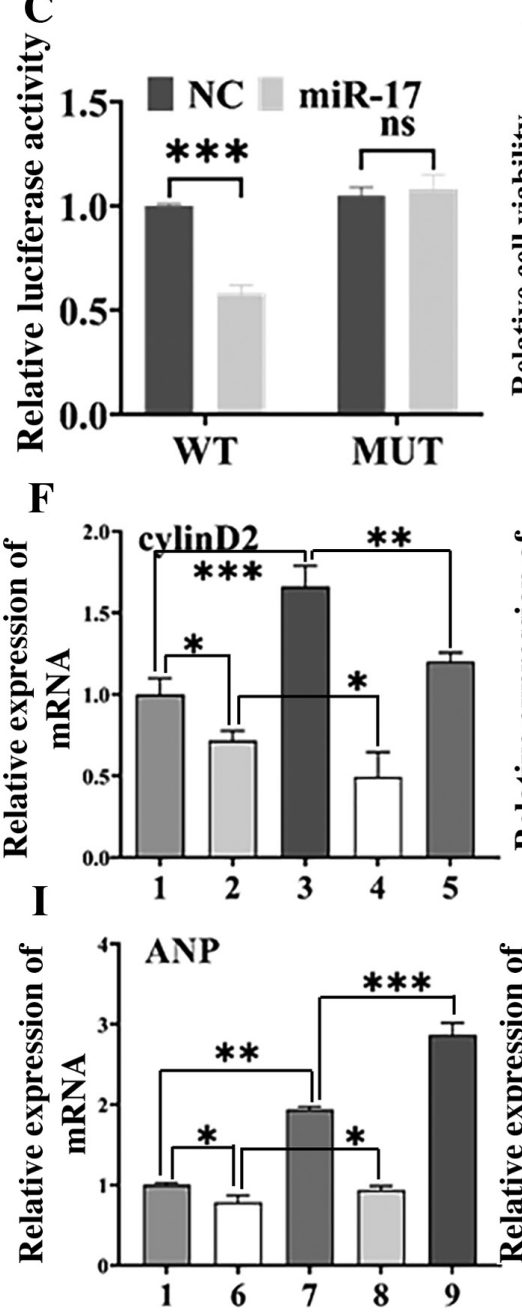

B

\section{5' accauuccuucgaaaaCGUGAAAg 3' cyclinD2 MUT}

\section{5' accauuccuucgaaaaGCACUUUg 3 ' cyclinD2 wT}

D

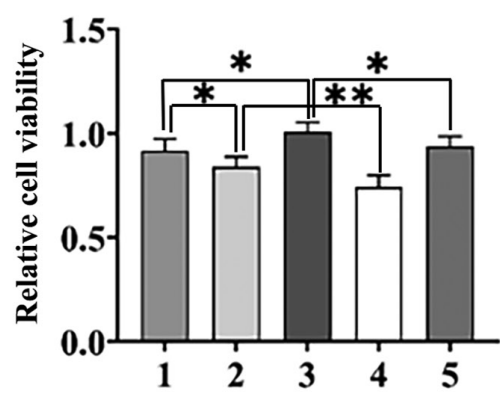

G

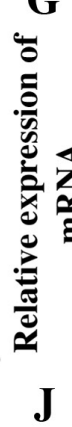

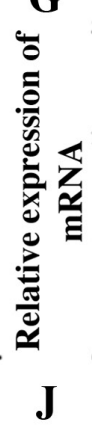

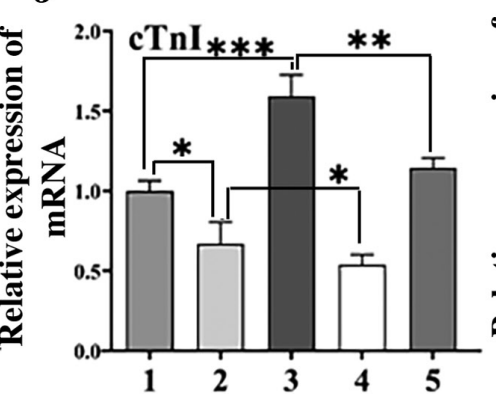

$\mathbf{E}$

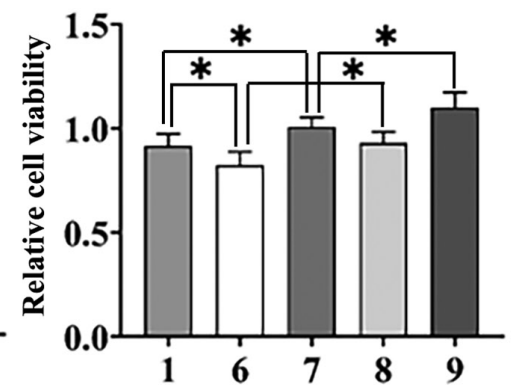

$\mathbf{H}$

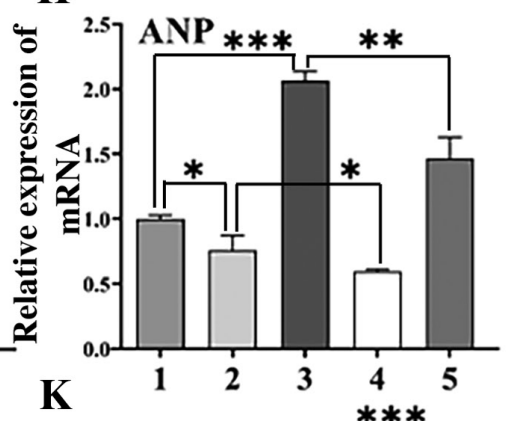

Figure 4. CyclinD2 is a direct target of miR-17 and hsa_circ_105039-mediated regulation of cell viability and differentiation is reversed by the restoration of cyclinD2, ANP and cTnI levels in DMSO-induced iPS cardiomyocytes. (A) Experimental grouping. (B) Sequence alignment of cyclinD2 and the potential binding site in the 3'-UTR of miR-17. (C) Luciferase reporter assay was performed in iPS cells co-transfected with the cyclinD2-WT plasmid or cyclinD2 plasmid. (D and E) 3-(4,5-dimethyl-2-thiazolyl)-2,5-diphenyl-2-H-tetrazolium bromide assay of iPS cell viability. Reverse transcription-quantitative PCR assay of the expression of (F and G) cyclinD2, (H and I) ANP and (J and K) cTnI. $\mathrm{n}=3$. ${ }^{*} \mathrm{P}<0.05,{ }^{* * *} \mathrm{P}<0.01$ and ${ }^{* * * *} \mathrm{P}<0.005$. ns, not significant; miR, microRNA; circ, circular RNA; ANP, atrial natriuretic peptide; cTnI, cardiac troponin I; iPS, induced pluripotent stem; DMSO, dimethyl sulfoxide; WT, wild-type; MUT, mutated; NC, negative control; si, small interfering RNA.

database predicted that cyclinD2 is a direct target of miR-17. The present study revealed the existence and functional importance of the hsa_circ_105039/miR-17/cyclinD2 axis in iPS cells for the first time, to the best of the authors' knowledge, and provided important insights into the role of this pathway in CHD. It showed that has_circRNA_105039 acted as a sponge for miR-17 to inhibit its regulation of
cyclinD2 transcription. These results indicated the protective functions of hsa_circ_105039 upregulation in iPS cells exposed to CHD.

In conclusion, by acting as a sponge for miR-17, hsa_circ_105039 may be a potential biomarker for prognosis and therapeutic target for CHD. Due to its functions including regulating the viability, migration, apoptosis and differentiation 
A

\section{Control}

2. si-NC+mimic

3. si-NC+inhibitor

4. si-circ_105039+mimic

5. si-circ_105039+inhibitor

6. $\mathrm{NC}+$ mimic

7. NC+inhibitor

8. circ_105039+mimic

9. circ $\_105039+$ inhibitor
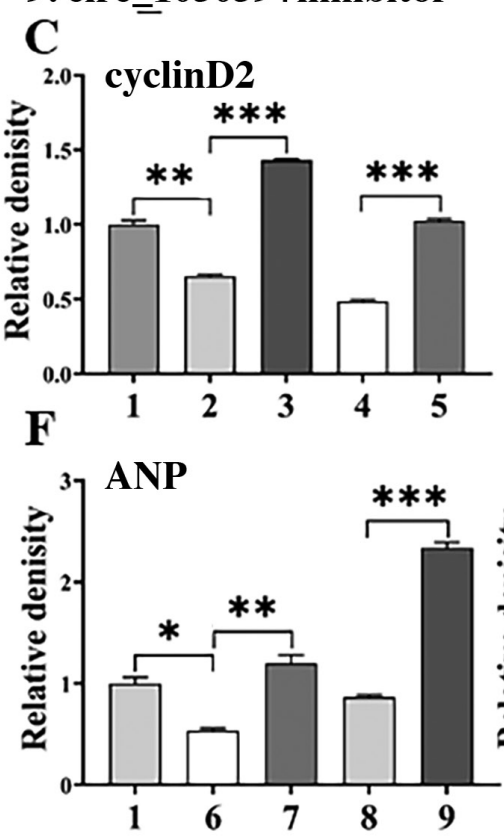

B
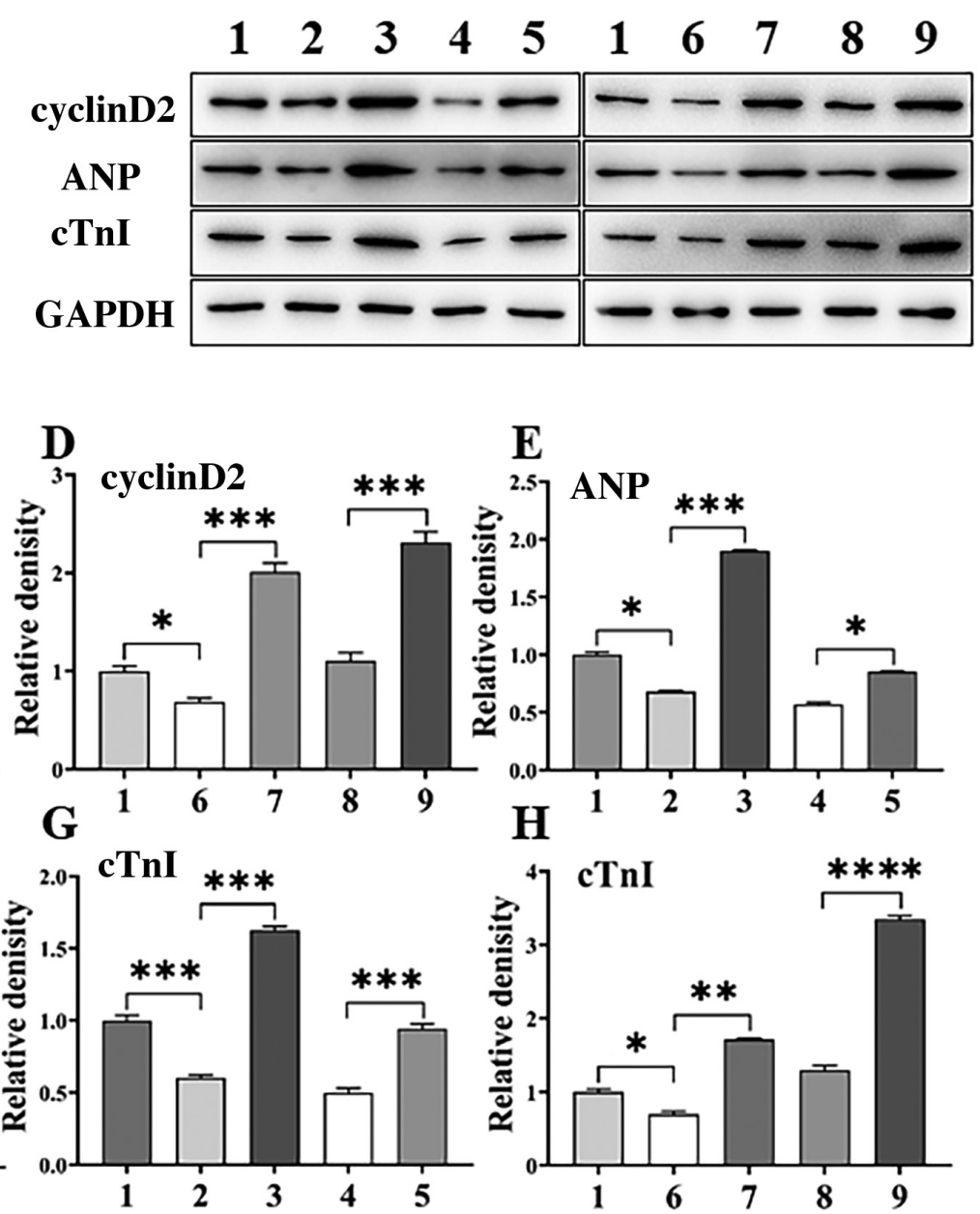

Figure 5. cyclinD2 is a direct target of miR-17 and hsa_circ_105039-mediated regulation of cell viability and differentiation is reversed by the restoration of cyclinD2, ANP and cTnI levels in DMSO-induced iPS cardiomyocytes. (A) Experimental grouping. (B) Western blotting bands. The analysis histogram of (C and D) cyclinD2, (E and F) ANP and (G and H) cTnI. $n=3 .{ }^{*} \mathrm{P}<0.05,{ }^{* *} \mathrm{P}<0.01,{ }^{* * *} \mathrm{P}<0.005$ and ${ }^{* * * *} \mathrm{P}<0.001$. ns, not significant; miR, microRNA; circ, circular RNA; ANP, atrial natriuretic peptide; cTnI, cardiac troponin I; iPS, induced pluripotent stem; NC, negative control; si, small interfering RNA.

of iPS cells, hsa_circ_105039 may be a potential key molecule in the diagnosis of CHD.

\section{Acknowledgements}

Not applicable.

\section{Funding}

The present study was supported by the National Natural Science Foundation of China (grant no. 81870240), Nanjing Municipality Health Bureau (grant no. JQX18010), Jiangsu Provincial Medical Youth Talent (grant no. QNRC2016114) and Foundation of Liaoning Province, China (grant no. 201602879).

\section{Availability of data and materials}

The datasets used and/or analyzed during the current study are available from the corresponding author on reasonable request.

\section{Authors' contributions}

BY performed the experiments and data analysis. ML conceived and designed the study and wrote the manuscript. SH performed the RT-qPCR and western blotting experiments, analyzed the related data, and reviewed and edited the manuscript. ZY and JZ designed the work, gave final approval of the version to be published, and agree to be accountable for all aspects of the work in ensuring that questions related to the accuracy or integrity of any part of the work are appropriately investigated and resolved. ZY and JZ confirm the authenticity of all the raw data. All authors read and approved the final manuscript.

Ethics approval and consent to participate

Not applicable.

Patient consent for publication

Not applicable. 


\section{Competing interests}

The authors declare that they have no competing interests.

\section{References}

1. Fernandes SM, Marelli A, Hile DM and Daniels CJ: Access and delivery of adult congenital heart disease care in the United States: Quality driven team based care. Cardiol Clin 38: 295-304, 2020.

2. Burchill LJ, Lee MGY, Nguyen VP and Stout KK: Heart failure in adult congenital heart disease. Cardiol Clin 38: 457-469, 2020

3. Schulkey CE, Regmi SD, Magnan RA, Danzo MT, Luther H, Hutchinson AK, Panzer AA, Grady MM, Wilson DB and Jay PY: The maternal-age-associated risk of congenital heart disease is modifiable. Nature 520: 230-233, 2015

4. Li Y, Klena NT, Gabriel GC, Liu X, Kim AJ, Lemke K, Chen Y, Chatterjee B, Devine W, Damerla RR, et al: Global genetic analysis in mice unveils central role for cilia in congenital heart disease. Nature 521: 520-524, 2015.

5. Bruneau BG: The developmental genetics of congenital heart disease. Nature 451: 943-948, 2008.

6. Sakhi R, Kauling RM, Theuns DA, Szili-Torok T, Bhagwandien RE, van den Bosch AE, Cuypers JAAE, Roos-Hesselink JW and Yap SC: Early detection of ventricular arrhythmias in adults with congenital heart disease using an insertable cardiac monitor (EDVA-CHD study). Int J Cardiol 305: 63-69, 2020

7. Meller CH, Grinenco S, Aiello H, Córdoba A, Sáenz-Tejeira MM, Marantz P and Otaño L: Congenital heart disease, prenatal diagnosis and management. Arch Argent Pediatr 118: e149-e161, 2020 (In English, Spanish)

8. Bishop L, Lansbury A and English K: Adult congenital heart disease and pregnancy. BJA Educ 18: 23-29, 2018.

9. Shum KK, Gupta T, Canobbio MM, Durst J and Shah SB: Family planning and pregnancy management in adults with congenital heart disease. Prog Cardiovasc Dis 61: 336-346, 2018.

10. Schmidt AB, Lund M, Corn G, Halldorsson TI, Øyen N, Wohlfahrt J, Olsen SF and Melbye M: Dietary glycemic index and glycemic load during pregnancy and offspring risk of congenital heart defects: A prospective cohort study. Am J Clin Nutr 111: 526-535, 2020.

11. Memczak S, Jens M, Elefsinioti A, Torti F, Krueger J, Rybak A, Maier L, Mackowiak SD, Gregersen LH, Munschauer M, et al: Circular RNAs are a large class of animal RNAs with regulatory potency. Nature 495: 333-338, 2013.

12. Hansen TB, Jensen TI, Clausen BH, Bramsen JB, Finsen B, Damgaard CK and Kjems J: Natural RNA circles function as efficient microRNA sponges. Nature 495: 384-388, 2013.

13. Jeck WR, Sorrentino JA, Wang K, Slevin MK, Burd CE, Liu J, Marzluff WF and Sharpless NE: Circular RNAs are abundant, conserved, and associated with ALU repeats. RNA 19: 141-157, 2013.

14. Costello A, Lao NT, Barron N and Clynes M: Reinventing the wheel: Synthetic circular RNAs for mammalian cell engineering. Trends Biotechnol 38: 217-230, 2020.

15. Verduci L, Strano S, Yarden $Y$ and Blandino G: The circRNA-microRNA code: Emerging implications for cancer diagnosis and treatment. Mol Oncol 13: 669-680, 2019.

16. Zhang Z, Yang T and Xiao J: Circular RNAs: Promising biomarkers for human diseases. EBioMedicine 34: 267-274, 2018

17. Bolha L, Ravnik Glavac M and Glavac D: Circular RNAs: Biogenesis, function, and a role as possible cancer biomarkers. Int J Genomics 2017: 6218353, 2017.

18. Huang Y, Zhang C, Xiong J and Ren H: Emerging important roles of circRNAs in human cancer and other diseases. Genes Dis 8: 412-423, 2020.

19. Thum T, Gross C, Fiedler J, Fischer T, Kissler S, Bussen M, Galuppo P, Just S, Rottbauer W, Frantz S, et al: MicroRNA-21 contributes to myocardial disease by stimulating MAP kinase signalling in fibroblasts. Nature 456: 980-984, 2008.

20. Liu N, Bezprozvannaya S, Williams AH, Qi X, Richardson JA, Bassel-Duby $\mathrm{R}$ and Olson EN: MicroRNA-133a regulates cardiomyocyte proliferation and suppresses smooth muscle gene expression in the heart. Genes Dev 22: 3242-3254, 2008.
21. Wu J, Li J, Liu H, Yin J, Zhang M, Yu Z and Miao H: Circulating plasma circular RNAs as novel diagnostic biomarkers for congenital heart disease in children. J Clin Lab Anal 33: e22998, 2019.

22. Clavijo PE, Friedman J, Robbins Y, Moore EC, Smith E, Zauderer M, Evans EE and Allen CT: Semaphorin4D inhibition improves response to immune-checkpoint blockade via attenuation of MDSC recruitment and function. Cancer Immunol Res 7: 282-291, 2019.

23. Livak KJ and Schmittgen TD: Analysis of relative gene expression data using real-time quantitative PCR and the 2(-Delta Delta C(T)) Method. Methods 25: 402-408, 2001.

24. Wada N, Wang B, Lin NH, Laslett AL, Gronthos S and Bartold PM: Induced pluripotent stem cell lines derived from human gingival fibroblasts and periodontal ligament fibroblasts. J Periodontal Res 46: 438-447, 2011.

25. Rowntree RK and McNeish JD: Induced pluripotent stem cells: Opportunities as research and development tools in 21st century drug discovery. Regen Med 5: 557-568, 2010.

26. Casiraghi A, Ardovino P, Minghetti P, Botta C, Gattini A and Montanari L: Semisolid formulations containing dimethyl sulfoxide and alpha-tocopherol for the treatment of extravasation of antiblastic agents. Arch Dermatol Res 299: 201-207, 2007.

27. Santos NC, Figueira-Coelho J, Martins-Silva J and Saldanha C: Multidisciplinary utilization of dimethyl sulfoxide: Pharmacological, cellular, and molecular aspects. Biochem Pharmacol 65: 1035-1041, 2003.

28. Kehat I, Kenyagin-Karsenti D, Snir M, Segev H, Amit M, Gepstein A, Livne E, Binah O, Itskovitz-Eldor J and Gepstein L: Human embryonic stem cells can differentiate into myocytes with structural and functional properties of cardiomyocytes. J Clin Invest 108: 407-414, 2001.

29. Inamdar MS, Venu P, Srinivas MS, Rao K and VijayRaghavan K: Derivation and characterization of two sibling human embryonic stem cell lines from discarded grade III embryos. Stem Cells Dev 18: 423-433, 2009.

30. Pal R, Mamidi MK, Das AK and Bhonde R: Diverse effects of dimethyl sulfoxide (DMSO) on the differentiation potential of human embryonic stem cells. Arch Toxicol 86: 651-661, 2012.

31. Behrens AN, Iacovino M, Lohr JL, Ren Y and Martin CM: Nkx2 5 mediates differential cardiac differentiation through interaction with Hoxa10. Stem Cells Dev 22: 2211-2220, 2013.

32. Cao N, Huang Y, Zheng J, Spencer CI, Zhang Y, Fu JD, Nie B, Xie M, Zhang M, Wang H, et al: Conversion of human fibroblasts into functional cardiomyocytes by small molecules. Science 352 : 1216-1220, 2016.

33. Wang Z and Huang J: Neuregulin-1 increases connexin-40 and connexin-45 expression in embryonic stem cell-derived cardiomyocytes. Appl Biochem Biotechnol 174: 483-493, 2014.

34. Behrens AN, Iacovino M, Lohr JL, Ren Y, Zierold C, Harvey RP, Kyba M, Garry DJ and Martin CM: Nkx2-5 mediates differential cardiac differentiation through interaction with Hoxa10. Stem Cells Dev 22: 2211-2220, 2013.

35. Cao N, Huang Y, Zheng J, Spencer CI, Zhang Y, Fu JD, Nie B, Xie M, Zhang M, Wang H, et al: Conversion of human fibroblasts into functional cardiomyocytes by small molecules, Science 352 : 1216-1220, 2016

36. Solé $\mathrm{C}$ and Lawrie $\mathrm{CH}$ : Circular RNAs and cancer: Opportunities and challenges. Adv Clin Chem 99: 87-146, 2020.

37. Sucharov CC, Miyamoto SD and Garcia AM: Circulating microRNAs as biomarkers in pediatric heart diseases. Prog Pediatr Cardiol 49: 50-52, 2018.

38. Kandettu A, Radhakrishnan R, Chakrabarty S, Sriharikrishnaa S and Kabekkodu SP: The emerging role of miRNA clusters in breast cancer progression. Biochim Biophys Acta Rev Cancer 1874: 188413, 2020.

39. Wu KJ: The role of miRNA biogenesis and DDX17 in tumorigenesis and cancer stemness. Biomedical Journal 43: 107-114, 2020.

40. Tesfaye AA, Azmi AS and Philip PA: miRNA and gene expression in pancreatic ductal adenocarcinoma. Am J Pathol 189: 58-70, 2019.

This work is licensed under a Creative Commons

Attribution-NonCommercial-NoDerivatives 4.0 International (CC BY-NC-ND 4.0) License. 